\title{
Consensus-Based ADMM for Distributed Optimization in Power Systems using Private Blockchain Network
}

\author{
Chinmay Shah, Member, IEEE, Jennifer King, Member, IEEE, Richard Wies, Senior Member, IEEE
}

\begin{abstract}
The optimization problem for scheduling distributed energy resources (DERs) and battery energy storage systems (BESS) integrated with power grid is important to minimize energy consumption from conventional sources in response to demand. Conventionally this optimization problem is solved in a centralized manner, which limits the size of the problem that can be solved, and also creates a high communication overhead since all the data is transferred to the central controller. These limitations are addressed by a proposed consensus-ADMM (alternating direction method of multiplier) based distributed optimization algorithm, which decomposes the optimization problem into sub-problems. The distribution feeder is partitioned into low coupling sub-networks/regions, which solves the subproblem locally and exchanges information with the neighboring regions to reach consensus to solve for the global update. The information exchange and synchronization between subnetworks/regions are vital for distributed optimization. In this work, both of these aspects are addressed by the blockchain. The smart contract deployed on the blockchain network acts as a virtual aggregator for synchronization in distributed computation. The blockchain-based distributed optimization problem's effectiveness is tested for 0.5-MW laboratory microgrid for one hour ahead and day-ahead for IEEE 123-bus and EPRI J1 test feeder, and results are compared with a centralized solution.
\end{abstract}

Index Terms - Alternating direction method of multiplier, Battery energy storage systems, Blockchain, Distributed optimization, Optimal power flow.

\section{INTRODUCTION}

$\mathrm{P}$ ower distribution networks are being reshaped by the integration of distributed energy resources (DERs) and battery energy storage systems (BESS) [1]. These DERs, when used intelligently, can reduce our dependence on conventional energy resources and thereby decrease the generation cost to meet the demand [2]. The optimal power flow (OPF) economic dispatch problem [3] seeks to optimize the objective of minimizing the total generation cost while satisfying the constraints imposed by the distribution network and device operating limits.

This work was supported in part by the U.S. Department of Energy with the Battelle Memorial Institute under Contract DEAC05-76RL01830 and under a subcontract Contract No. 474633 from Pacific Northwest National Laboratories to the University of Alaska Fairbanks.

C. Shah is with the Department of Electrical and Computer Engineering, College of Engineering and Mines, University of Alaska Fairbanks, Fairbanks, AK 99775-5915 USA (e-mail: cshah@alaska.edu).
The OPF problem is challenging to solve because of the nonconvex feasible set, and hence convex relaxation would ease the computation complexity [4]. The second-order cone program (SOCP) relaxation for the power distribution network is proposed in [5]-[9]. The SOCP relaxed OPF problem can be solved using any convex optimization solver.

The authors of [10]-[15] have proposed algorithms for optimal power flow in power distribution networks with integrated BESS but are solved centrally. A centralized approach to solve the OPF problem in power distribution networks with a large number of integrated DERs and BESS is computationally challenging and exhibits poor scalability. A distributed optimization algorithm can address this challenge.

Several distributed optimization algorithms have been adapted and/or developed in recent years to solve the OPF problem, which decomposes the central problem into local subproblems. The algorithm in [16] is based on dual decomposition method and in [17]-[18] on the auxiliary variable method. The alternating direction method of multiplier (ADMM) [19], which is simultaneously general and scalable, is exploited in most of the recent research work to solve the OPF problem in a distributed fashion. The ADMM algorithm in [20]-[21] solves the OPF problem, exchanging voltage information between the neighboring regions; however, DERs and BESS are not integrated into the test network. The OPF problem with nonconvex feasible sets in [4], [23], and [24] is solved using a decentralized algorithm in which a specific node communicates only with its adjacent nodes with no central aggregator. The limitation of [4], and [23] is that the synchronization will not be easily achieved between the local sub-problems without the central aggregator. The other limitation is the exchange of information between each node in the network during the distributed computation, which can be exhaustive in a bigger system.

The communication infrastructure is essential for information exchange between the neighboring regions/nodes or the central aggregator to solve the distributed optimization problem. As discussed in [25], there are two kinds of

J. King is with the National Renewable Energy Laboratory, Golden, CO 80401 USA (e-mail: Jennifer.King@nrel.gov).

R. W. Wies, is with the Department of Electrical and Computer Engineering, College of Engineering and Mines, University of Alaska Fairbanks, Fairbanks, AK 99775-5915 USA (e-mail: rwwiesjr@alaska.edu). 
communications infrastructure: 1) Centralized infrastructure and 2) Distributed infrastructure. The SCADA system is an example of centralized infrastructure, where local devices or RTUs send the data to the central controller and cannot exchange information amongst each other. The limitation of centralized infrastructure is that the fault in the central controller can halt the entire network. With the recent developments in IoT (internet of things) technology, the local controllers and RTUs (remote terminal units) can communicate with each other [26] and is an example of distributed communication infrastructure. The limitation of distributed infrastructure, without the central controller, is the synchronization of the local sub-problems.

In our work, the limitations mentioned above are addressed by deploying a smart contract on a blockchain network which acts as a virtual aggregator. Blockchain technology enables the direct and reliable exchange of information between the participating nodes or assets by establishing a secure and tamper-proof digital platform [27]-[28]. The blockchain has been used in [24] to store the optimal schedule and to make automatic and secure payments.

The main objective of this research work is to implement a distributed general form consensus OPF problem on a blockchain platform, which is solved by the ADMM-based method. The novel contributions of this research work are as follows:

1) The proposed consensus ADMM based OPF algorithm is implemented for distribution feeder partitioned into low coupling sub-networks/regions. The information exchange between the sub-networks instead of each node reduces the computation time, and the algorithm converges quickly.

2) The proposed consensus ADMM based OPF algorithm on the blockchain network utilizes the smart contract as a virtual aggregator, which provides secure data exchange, and synchronization between the local controllers of the sub-networks to solve the global update. Advanced distribution management systems could deploy smart contracts in existing utility grids.

3) The proposed consensus ADMM based OPF algorithm is tested for the Alaska Center for Energy and Power (ACEP) 0.5 MW Power System Integration (PSI) Laboratory Microgrid [29].

The rest of the paper is organized as follows. Section II describes the formulation of the OPF problem, BESS model, blockchain, and smart contract. The consensus ADMM method implemented on the blockchain network is explained in section III, followed by the test feeder network formulation in section IV. The simulation results are presented in section V. Section VI concludes the paper and discusses future work followed by acknowledgments.

\section{FoRMULATION OF OPF PROBLEM}

In this section, we define an OPF problem for the power distribution network. The distribution network includes a substation node, renewable energy resources, fixed loads, BESS, and electric vehicles (EVs) as shapeable loads [30].

\section{A. Distribution Network Model}

The radial graph distributed network model $\check{\mathrm{R}}:=(\mathrm{N}, \varepsilon)$ where $N:=\{0,1,2 \ldots, n\}$ represents the set of nodes, and $\varepsilon$ represents the set of distribution lines connecting these nodes in the network. $\mathrm{N}=0$ represents the root node, and the other nodes in $\mathrm{N}$ represent the branch nodes. For each node $i$, it has a unique parent node $A_{i}$ and a set of children nodes $C_{i}=\left[j_{i}, j_{2}, \ldots, j_{k}\right]$ [4]. For each line that connects node $i$ to its parent node $A_{i}$, let $z_{i}=r_{i}+j x_{i}$ be the impedance of the line.

For each node $i \in \mathrm{N}$, let $V_{i}$ be the complex voltage, $v_{i}=$ $\left|V_{i}\right|^{2}$, and $s_{i}=p_{i}+j q_{i}$ be the net complex power injection. Let $I_{i}$ be the complex current flowing from node $i$ to parent node $A_{i}, l_{i}=\left|I_{i}\right|^{2}$, and $S_{i}=P_{i}+j Q_{i}$ be the complex power flowing from node $i$ to child node $C_{i}$. The branch flow model for this radial network is given by:

$$
\begin{gathered}
p_{i}=P_{i}-\sum_{k \in C_{i}} P_{k}+r_{i} l_{i}, i=0,1, \ldots, n \\
q_{i}=Q_{i}-\sum_{k \in C_{i}} Q_{k}+x_{i} l_{i}, i=0,1, \ldots, n \\
v_{i}=v_{A_{i}}+2\left(r_{i} P_{i}+x_{i} Q_{i}\right)-\left(r_{i}{ }^{2}+x_{i}{ }^{2}\right) l_{i}, i=1, \ldots, n \\
\left|S_{i}\right|^{2}=v_{i} l_{i}, i=1, \ldots, n
\end{gathered}
$$

\section{B. Network Components}

The set of network components at a particular node $i$ is denoted as:

$p_{i}^{g}$ - Diesel generator/substation active power

$p_{i}^{p v}$ - Photovoltaic (PV) generator unit active power

$p_{i}^{\text {wind }}$ - Wind turbine active power

$p_{i}^{b-c h}$ - BESS (charging) active power

$p_{i}^{b-d c h}$ - BESS (discharging) active power

$p_{i}^{f}$ - Fixed load

$p_{i}^{S}$ - Shapeable load (Electric Vehicles-EVs)

$q_{i}^{g}$ - Diesel generator/substation reactive power

$q_{i}^{f}$ - Fixed load (reactive)

The net active and reactive power injection at a particular node $i$ is given by:

$$
\begin{gathered}
p_{i}=p_{i}^{g}+p_{i}^{p v}+p_{i}^{w i n d}+p_{i}^{b-d c h}-p_{i}^{b-c h}-p_{i}^{f}-p_{i}^{S} \\
q_{i}=q_{i}^{g}-q_{i}^{f}
\end{gathered}
$$

\section{Battery Energy Storage System (BESS) Model}

The BESS is characterized by its rated power $\left(p_{i}^{b}\right)$, rated energy $\left(E_{i}^{b}\right)$, and charging/discharging cycle efficiencies $\left(\eta_{c h}\right.$ and/or $\left.\eta_{d c h}\right)$. The BESS model that is implemented in this paper is a mixed-integer linear model [31]. The charge and discharge state in the model is formulated as:

$$
\begin{gathered}
p_{i}^{b-n e t}(t)=\eta_{c h} p_{i}^{b-c h}(t)-\left(\frac{1}{\eta_{d c h}}\right) p_{i}^{b-d c h}(t) \\
E_{i}^{b-\min } \leq E_{i 0}^{b}+\Delta t \sum_{k=1}^{t} p_{i}^{b-n e t}(k) \leq E_{i}^{b-\max } \\
0 \leq p_{i}^{b-c h}(t) \leq p_{i}^{b-c h-\max }
\end{gathered}
$$




$$
0 \leq p_{i}^{b-d c h}(t) \leq p_{i}^{b-d c h-\max }
$$

where $i \in \mathrm{N}, t=0,1,2, \ldots, T-1$, and $T=24 \mathrm{~h}$.

\section{Centralized OPF and SOCP Relaxation of Constraints}

The thermal generator cost at the substation node is typically represented by a quadratic fuel cost function given by:

$$
f_{i}\left(p_{i}^{g}(t)\right)=\alpha_{i}\left(p_{i}^{g}(t)\right)^{2}+\beta_{i} p_{i}^{g}(t)+\gamma_{i}(t)
$$

The optimal power flow (OPF) problem aims at minimizing the generation cost subject to branch flow equations and local DER constraints and is formulated as,

$$
\begin{gathered}
\min \sum_{t=1}^{T} \sum_{i=0}^{N} f_{i}\left(p_{i}^{g}(t)\right) \\
\text { s.t. }(1 a),(1 b),(1 c),(1 d) \quad t=1,2, \ldots, T \\
\underline{v_{i}} \leq v_{i}(t) \leq \overline{v_{i}} \\
\text { solve BESS constraints }(3 \mathrm{a}-3 \mathrm{~d}) \\
p_{i}^{s-\min } \leq p_{i}^{S}(t) \leq p_{i}^{s-\max } \\
\sum_{t=1}^{T} p_{i}^{S}(t)=E_{i, \text { dem }}^{s} \\
p_{i}^{S}(t)=0 \quad \forall t=1,2, \ldots, t_{\text {start }} \\
p_{i}^{s}(t)=0 \quad \forall t=t_{s t o p}, \ldots, T \\
\text { over } p_{i}^{g}(t) \epsilon\left[\overline{p_{l}^{g}}, \underline{p_{i}^{g}}\right], q_{i}^{g}(t) \epsilon\left[\overline{q_{l}^{g}}, \underline{q_{i}^{g}}\right], i=0, \ldots, N
\end{gathered}
$$

where $p_{i}^{g}(t)$ is the decision variable, and $E_{i \text {, dem }}^{S}$ is the net energy demand of the shapeable load (e.g., EV) and is charged between times $t_{\text {start }}$ and $t_{\text {stop }}$.

The quadratic equality constraint in (1d) makes the above OPF problem non-convex. The OPF problem can be relaxed to a convex problem by SOCP relaxation of (1d) given by,

$$
\left|S_{i}\right|^{2} \leq v_{i} l_{i}, i=1, \ldots, N
$$

The selection of model-1 for the BESS yields a mixed-integer linear OPF problem (MILP), whereas model-2 yields a mixedinteger convex quadratic OPF problem (MICQP), and model-3 gives us a mixed-integer nonlinear OPF problem (MINLP).

\section{E. Blockchain and Smart Contracts}

The blockchain is a prominent technology that provides a secure and distributed database that contains the log of transactions in chronological order. The digital users/nodes on this blockchain network communicate through the Peer-to-Peer (P2P) system to reach a consensus on the valid state of the ledger. This is contrary to centralized systems which need the central authority to act as the intermediary to maintain the database and transactions and guard its authenticity. Furthermore, the security of the blockchain network is enhanced by hash functions and public-key cryptography and depends on the type of network, i.e., public or private network [32]. The users can either join the public blockchain network or the private blockchain network. In this research work, private network with the permissioned ledger is used that has reduced security but is fast, easy to scale, and energy-efficient [33].

The full potential of blockchain technology can only be utilized using smart contracts. The smart contract is a userdefined executable program that can alter the ledger and perform specific tasks when called upon by the users. The Ethereum is an open-source programmable distributed computing platform with smart contract functionality [34]. The distributed OPF algorithm proposed in this paper is developed on the private Ethereum network in which the smart contract is deployed to act as a virtual aggregator. The block diagram of the private Ethereum network for distributed OPF problem is shown in Fig. 1. The workflow showing the interaction between the smart contract and the users while solving the distributed OPF problem is explained in the following section.

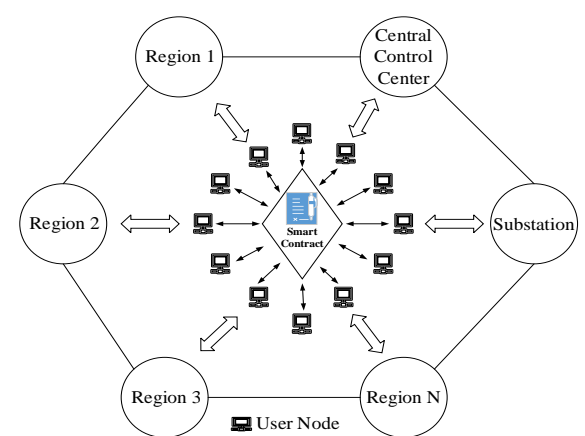

Fig. 1. Ethereum blockchain architecture for distributed OPF problem.

\section{DISTRIBUTED OPF PROBLEM FORMULATION}

In this section, the distributed optimization algorithm based on consensus ADMM is presented to solve the OPF problem. Once the solution method is established, the algorithm is implemented on the blockchain network.

\section{A. General Form Consensus ADMM}

The general form consensus optimization problem is expressed by

$$
\min _{\text {s.t. } x_{i}-z_{i}=0, i=1,2,3, \ldots, N} \sum_{i=1}^{N} f_{i}\left(x_{i}\right)
$$

where $x_{i} \in R^{n_{i}}, i=1,2,3, \ldots, N$ is a local variable which is related to the subproblem and consists of a selection of the components of the global variable $z \in R^{N}$. The mapping from local variable indices to some global variable index is expressed as $g=\mathrm{G}(i, j)$, which means that the local variable component $\left(x_{i}\right)_{j}$ corresponds to the global variable component $z_{g}$ [23]. Now, for the ease of notation, let $\widetilde{z_{l}} \in R^{N_{i}}$ be defined by $\left(\tilde{z}_{i}\right)_{j}=z_{\mathrm{G}(i, j)}$, representing the approximate mapping of the local variable $x_{i}$ into the global variable. The consensus constraint can now be expressed as $x_{i}-\widetilde{z_{l}}=0$.

The augmented Lagrange function for the problem in (7) is $L(x, z, u)=\sum_{i=1}^{N}\left(f_{i}\left(x_{i}\right)+u_{i}^{T}\left(x_{i}-\widetilde{z_{l}}\right)+\left(\frac{\rho}{2}\right)\left\|x_{i}-\widetilde{z}_{l}\right\|_{2}^{2}\right)(8)$ where the dual variable $u_{i}^{T} \in R^{N_{i}}$, and $\rho$ is the penalty parameter. The iterations to solve the general form consensus ADMM problem mentioned in (8) is given by 


$$
\begin{gathered}
x_{i}^{n+1}=\underset{x_{i}}{\operatorname{argmin}}\left(f_{i}\left(x_{i}\right)+u_{i}^{T}\left(x_{i}\right)+\left(\frac{\rho}{2}\right)\left\|x_{i}-\widetilde{z}_{l}\right\|_{2}^{2}\right) \\
z^{n+1}=\underset{z}{\operatorname{argmin}}\left(\sum_{i=1}^{N}-u_{i}^{T}\left(\tilde{z}_{i}\right)+\left(\frac{\rho}{2}\right)\left\|x_{i}^{n+1}-\widetilde{z}_{l}\right\|_{2}^{2}\right) \\
u_{i}^{n+1}=u_{i}^{n}+\rho\left(x_{i}^{n+1}-z_{i}^{n+1}\right)
\end{gathered}
$$

where $x_{i}$ and $u_{i}$ updates can be carried out separately and locally in parallel for each iteration $n$.

The $z$-update step decouples across the components of $z$ and is expressed by

$$
z_{g}^{n+1}=\frac{\sum_{\mathrm{G}(i, j)=g}\left(\left(x_{i}^{n+1}\right)_{j}+\left(\frac{1}{\rho}\right)\left(u_{i}^{n}\right)_{j}\right)}{\sum_{\mathrm{G}(i, j)=g^{1}}}
$$

where $z_{g}$ is obtained by averaging all the elements of $x_{i}^{n+1}+$ $\left(\frac{1}{\rho}\right) u_{i}^{n}$ that corresponds to the global index $g$. Now, after the first iteration for consensus $\mathrm{ADMM}, \sum_{\mathrm{G}(i, j)=g}\left(y_{i}^{n}\right)_{j}=0$, i.e., the sum of the dual variable entries that correspond to global index $g$ is zero. The $z$-update step can now be expressed as

$$
z_{i}^{n+1}=\left(\frac{1}{k_{g}}\right) \sum_{\mathrm{G}(i, j)=g}\left(x_{i}^{n+1}\right)_{j}
$$

where $k_{g}$ is the number of local variable entries that correspond to the global variable entry $z_{g}$.

The primal and dual residuals for the general form consensus ADMM is defined by

$$
r^{n}=x_{i}^{n}-\bar{z}_{i}^{n}, \quad s^{n}=-\rho\left(z^{n}-z^{n-1}\right)
$$

The consensus ADMM converges to an optimal solution when the following condition is met:

$$
\left\|r^{n}\right\|_{2} \leq \epsilon_{\text {primal }}, \text { and }\left\|s^{n}\right\|_{2} \leq \epsilon_{\text {dual }}
$$

where $\epsilon_{\text {primal }}$ and $\epsilon_{\text {dual }}$ are the tolerances for the primal and dual residuals.

\section{B. Application of General Consensus ADMM to OPF}

To solve the distributed OPF problem, we partition the distribution network into sub-networks/regions, as shown in Fig. 2. Now the OPF problem for the entire distribution network is decomposed into global consensus form using the ADMM based method in such a way that each region needs to solve its local sub-problem in each iteration. The local sub-problem has its own objective function and set of constraints and is formulated for a particular region $i$ as

$$
\min \sum_{t=1}^{T} f_{i}\left(p_{i}^{g}(t)\right)
$$

$$
\text { Subject to (5b) - (5i). }
$$

The local variables to solve the sub-problem presented in (16) for a particular region $i$ is denoted by $x_{i}=$ $\left[p_{i}, q_{i}, p_{i}^{g}, q_{i}^{g}, p_{i}^{P V}, p_{i}^{\text {wind }}, p_{i}^{b-c h}, p_{i}^{b-d c h}, p_{i}^{f}, q_{i}^{f}, p_{i}^{s}\right]$ and is not shared over the network. The set of coupling local variables to exchange information between different regions in the network is denoted by $\tilde{x}_{i}=\left[P_{i}, Q_{i}, v_{i}, P_{C_{i}, i}, Q_{C_{i}, i}, v_{A_{i}, i}\right]$. The global variable index that maps the local coupling variable is $g=$ $\mathrm{G}(i, j)$, i.e., $\tilde{x}_{i}=z_{\mathrm{G}(i, j)}$. Let $\tilde{z}_{i}=[P, Q, v] \in R^{N}$ be defined by $\left(\tilde{z}_{i}\right)_{j}=z_{\mathrm{G}(i, j)}=z_{g} . \tilde{z}_{i}$ is the subset of the global variable $z=$ $[P, Q, v] \in R^{3 N}$. To reach the consensus between the local and the global variable for all the regions, $\tilde{x}_{i}-\tilde{z}_{i}=0$.

The augmented Lagrangian for the OPF problem defined in (18) is given by

$$
\left.L_{\rho}=\sum_{t=1}^{T} f_{i}\left(p_{i}^{g}(t)\right)+u_{i}^{T}\left(\tilde{x}_{i}-\widetilde{z_{l}}\right)+\left(\frac{\rho}{2}\right)\left\|\tilde{x}_{i}-\widetilde{z}_{\imath}\right\|_{2}^{2}\right)
$$

This is decomposed into the general form consensus ADMM, and each region $i$ is locally solved using (18) subject to (5b) (5i).

$\left.\tilde{x}_{i}^{n+1}=\min \sum_{t=1}^{T} f_{i}\left(p_{i}^{g}(t)\right)+u_{i}^{T}\left(\tilde{x}_{i}\right)+\left(\frac{\rho}{2}\right)\left\|x_{i}-\widetilde{z}_{l}\right\|_{2}^{2}\right)$

where $\tilde{z}_{i}$ is received from the aggregator.

The region $i$ then sends the new $\tilde{x}_{i}^{n+1}$ to the aggregator, who computes the following $z$-update for each individual global variable $z_{g}^{n+1}$

$$
z_{g}^{n+1}=\left(\frac{1}{k_{g}}\right) \sum_{\mathrm{G}(i, j)=g}\left(\tilde{x}_{i}^{n+1}\right)_{j}
$$

The regions then receive the new $z_{g}^{n+1}$ and update the dual variable as follows:

$$
u_{i}^{n+1}=u_{i}^{n}+\rho\left(\tilde{x}_{i}^{n+1}-\tilde{z}_{i}^{n+1}\right)
$$

The convergence criteria for the algorithm are defined in (14) and (15) with the values for $\epsilon_{\text {primal }}$ and $\epsilon_{\text {dual }}$ set to $1 \mathrm{e}-5$.

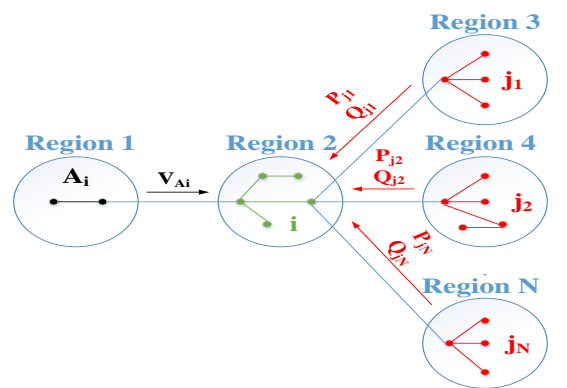

Fig. 2. General consensus ADMM for OPF in a distribution network

\section{Implementing the Algorithm on Blockchain Network}

The private Ethereum blockchain network is used to implement the distributed OPF algorithm for the distribution network in this research work. The go-ethereum client (geth) is used to run a full Ethereum node. Each node/user on the Ethereum network is assigned a personal account defined by a private and public key. These accounts are indexed by their address ( $\Pi$ ) derived from the public key. There are two types of Ethereum accounts: 1) externally owned accounts (EOAs) and 2) contracts accounts. The EOA is controlled by a private key and can transfer ether or trigger a contract code. The contract account has its own code and is controlled by that code. Upon establishing the Ethereum network to implement the 
consensus ADMM for the distributed OPF problem, an externally owned account indexed by address $\Pi_{i}$ is set up for every region $i$ in the distribution network. The contracts account indexed by address $\Psi$ is set up for the control center that deploys the smart contract (modeled in Solidity [35]) to the network. The address $\Psi$ is shared with all regions $i$ in the network to communicate with the smart contract. The users/nodes interact with the Ethereum network by sending a transaction from an account by using a web3 object that connects to a JSON-RPC server [36]. The steps of the consensus ADMM for the distributed OPF problem implemented on the Ethereum network are expressed below.

\section{Algorithm: Consensus ADMM for distributed OPF problem executed} on the Blockchain network.

1. $\tilde{z}_{i}=0, u_{i}=0, \rho=0.5, \epsilon_{\text {primal }} 1 e-5$, and $\epsilon_{\text {dual }}=1 e-5$ are initialized on the smart contract.

2. Every region $i$ establishes a connection with the smart contract and retrieve the values of $\tilde{z}_{i}, u_{i}$, and $\rho$.

3. The local optimization problem in (18) is solved by each region $i$ and the updated values of the coupling variable $\tilde{x}_{i}$ is submitted to the smart contract.

4. When all the regions have submitted their coupling variable, the smart contract will execute the z-update for each individual global variable $z_{g}^{n+1}$ as expressed in (19).

5. After step 4 is complete, the smart contract will send the updated values of the global variable to the regions $i$ to calculate $u_{i}$ described in (20).

6. The region $i$ will calculate the primal and dual residual values (14).

7. Each region $i$ will send the updated values of $u_{i}, r^{n}$, and $s^{n}$ to the smart contract. The contract will check for the stopping criteria $\left\|r^{n}\right\|_{2} \leq \epsilon_{\text {primal }}$ and $\left\|s^{n}\right\|_{2} \leq \epsilon_{\text {dual }}$. If the stopping criteria are not met, the problem proceeds to step 2; otherwise, STOP.

\section{CASE STUDY}

In this section, we discuss about validating the centralized and proposed consensus ADMM distributed OPF algorithms for the 0.5 MW Alaska Center for Energy and Power (ACEP) Power System Integration (PSI) lab microgrid with four control nodes and implementing the same for the IEEE 123-bus and EPRI J1 test feeder system.

\section{A. ACEP PSI Laboratory Microgrid}

The ACEP PSI lab operates on the same scale as one of Alaska's village power systems and can be modified for individual test scenarios. The PSI lab infrastructure includes a $320 \mathrm{~kW}$ diesel generator, a $100 \mathrm{~kW}$ wind emulator, a 540 VDC/1000 A-hr lead-acid battery, $312 \mathrm{kVA}$ inverter, and two $250 \mathrm{~kW}$ programmable load banks. The one-line diagram of the ACEP PSI lab is available in [29].

\section{B. IEEE 123-Bus Test Network}

The IEEE 123-bus test feeder used in this study consists of 114 branch buses and is partitioned into seven regions, as shown in Fig. 3. The test feeder is also modified to a balanced network. The nominal operating voltage of the test feeder is $4.16 \mathrm{kV}$. The consensus ADMM optimization algorithm in (18), (19), and (20) is implemented for the partitioned IEEE 123-bus, where each region solves its local sub-problem and exchanges information with the neighboring regions.

Region 1 is a root/reference bus, which can either be a substation or thermal/diesel generator with a quadratic fuel cost function. Other regions consist of branch nodes that have a deterministic load profile, rooftop PV, shapeable load (EV), and BESS. The shapeable loads and BESS at each node are half the peak residential load. The storage capacity of BESS is 6 hours.

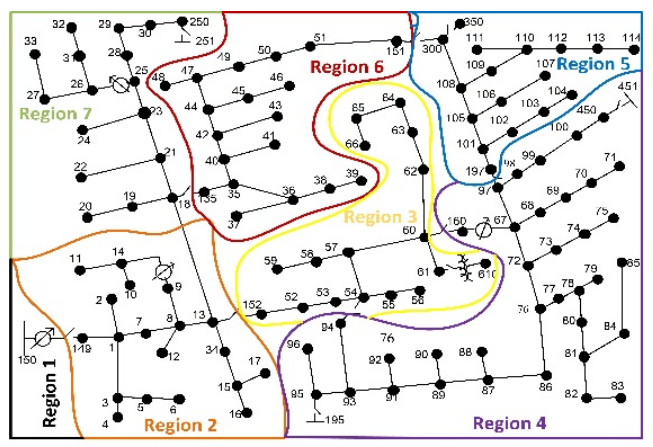

Fig. 3. IEEE 123-bus test feeder partitioned into 7 regions.

The PECAN STREET project residential load data set from Jan 1 to June 30, 2017, is used in this research work [37]. Based on the results of the load profile aggregation (LPA) algorithm proposed in [38] for the IEEE 123-bus test feeder, there are average 8 houses on each bus with $3.5 \mathrm{~kW}$ peak load/house. The capacity of the rooftop PV installed at each house on branch nodes is $6 \mathrm{~kW}$. The hourly 2017 NSRDB solar data from Jan 1 to June 30, 2017, is used for the rooftop PV.

\section{EPRI J1 Test Feeder}

The EPRI J1 test feeder has 3434 buses which serves approximately 1300 residential, commercial, and light industrial customers via 58 miles of $12.47 \mathrm{kV}$ primary line [39]. The feeder is modified to a balanced three phase network and is partitioned into seven regions as shown in Fig. 4 for this work.

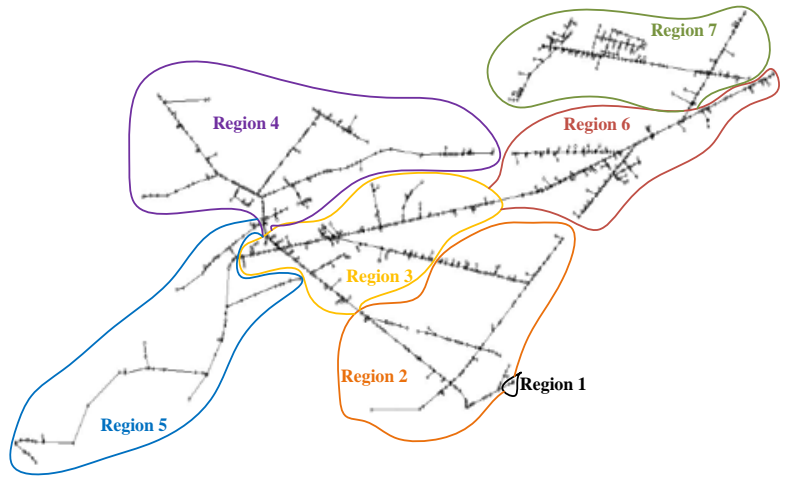

Fig. 4. EPRI J1 feeder partitioned into 7 regions.

The region 1 is a substation, whereas other regions consist of branch nodes that have residential, commercial, and light industrial load, residential roof-top PV, customer owned large PV system, shapeable loads (EVs), and BESS. The peak load of the entire feeder is approximately $6 \mathrm{MW}$. The shapeable load at each node with residential load is half the peak load. The 1.7 MW customer-owned PV systems already exist on the feeder. Additionally, rooftop PV and BESS rated 50\% of the daily consumption for each residential load is also considered in this research work. The storage capacity of BESS is 6 hours. The hourly June 2012 solar data from EPRI [39] is used for PV.

\section{Implementing the Test System on Ethereum Network}

The go-ethereum/Geth client is installed for running the ethereum node on the computer and is used to connect to the 
private ethereum network. Upon setting up the network, the smart contract to implement the virtual aggregator for the consensus ADMM OPF problem for the ACEP PSI lab, IEEE 123-bus, and EPRI J1 test feeder is developed and compiled in Solidity. The local sub-problems are executed using Python and CVXPY. After solving the local sub-problems, each region in the ACEP PSI lab, IEEE 123-bus, and EPRI J1 test feeder communicates with Ethereum network using web3.py.

\section{RESULTS}

\section{A. ACEP PSI Lab}

The centralized OPF algorithm was tested in the ACEP PSI lab using a spinning reserve control (SRC) strategy [29]. The SRC maintains the loading on the diesel-electric generator between the minimum and maximum set points by using the inverter to compensate for over or under loading conditions. The OPF algorithms was simulated for 1 hour with a time step of 2.5 minutes. The dispatch plan generated by the algorithm and the results from the lab are presented in Fig. 5 to Fig. 8.

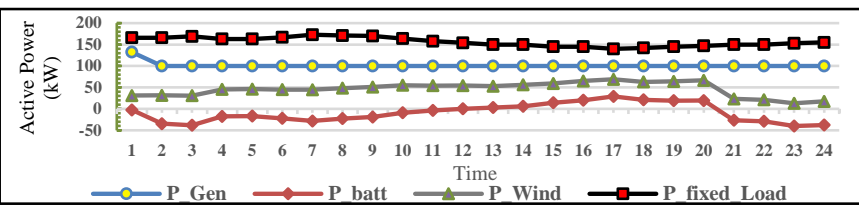

Fig. 5. Dispatch plan for ACEP PSI lab generated by centralized OPF algorithm.

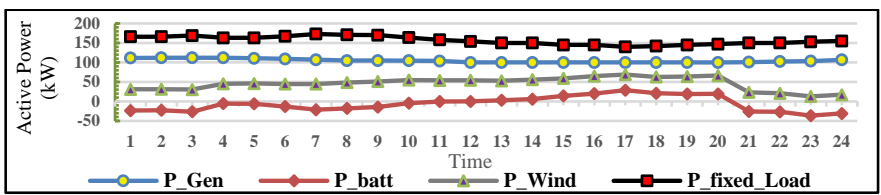

Fig. 6. Dispatch plan for ACEP PSI lab generated by distributed consensus ADMM OPF algorithm.

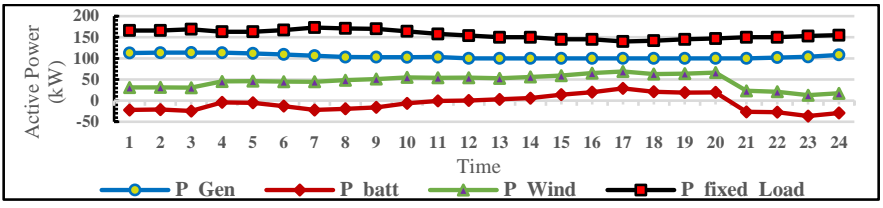

Fig. 7. Dispatch plan for ACEP PSI lab generated by distributed consensus ADMM OPF algorithm implemented on the blockchain network.

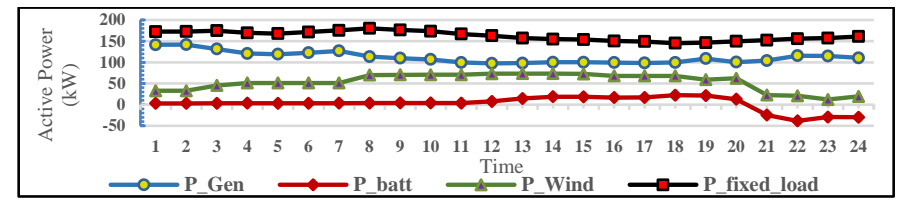

Fig. 8. ACEP PSI lab results.

We can observe from the results that the battery energy storage discharges to the net load and charges from the excessive wind power. The minimum generation from the diesel generator was set at $100 \mathrm{~kW}$ to prevent wet stacking.

\section{B. IEEE 123-Bus and EFRI J1 Test Feeder}

The centralized algorithm was tested using the IEEE 123-bus test feeder, and the results are published in [30]. The dispatch plan generated for the IEEE 123-bus and EPRI J1 test feeder using the centralized MILP algorithm is shown in Fig. 9, and Fig. 10 respectively; and using distributed consensus ADMM w/o and with the blockchain are shown from Fig. 11 to Fig. 14.

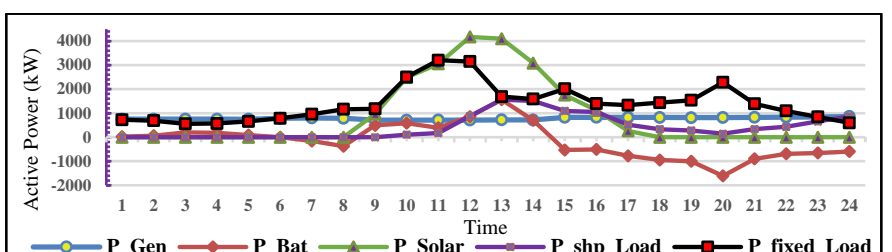

Fig. 9. Dispatch plan for the IEEE 123-bus test feeder generated by a centralized MILP OPF algorithm.

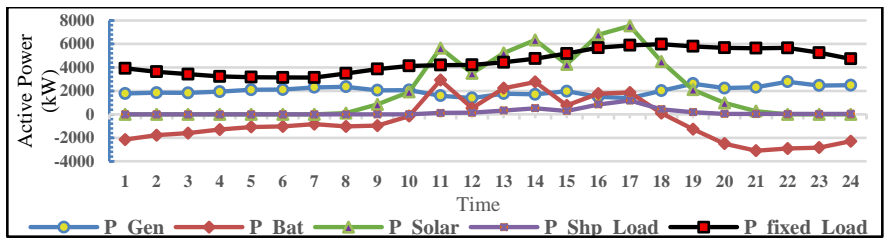

Fig. 10. Dispatch plan for the EPRI J1 test feeder generated by a centralized MILP OPF algorithm.

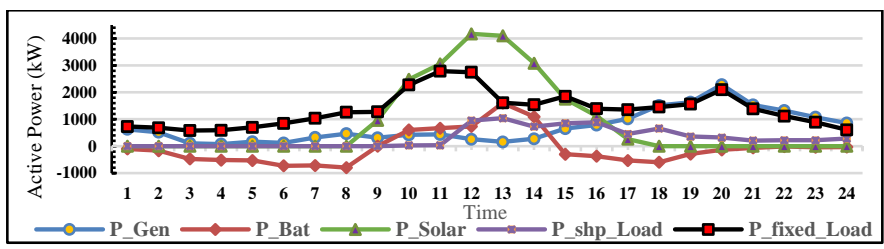

Fig. 11. Dispatch plan for the IEEE 123-bus test feeder generated by distributed consensus ADMM OPF algorithm.

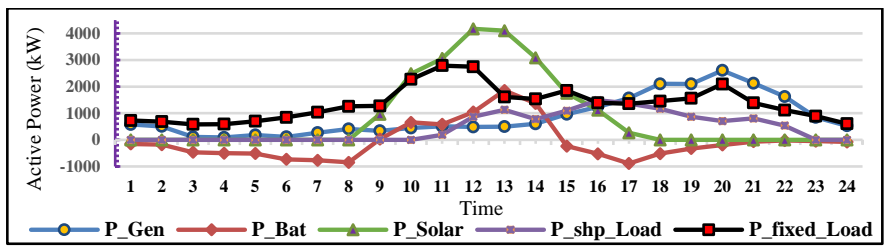

Fig. 12. Dispatch plan for IEEE 123-bus test feeder generated by distributed consensus ADMM OPF algorithm implemented on the blockchain network.

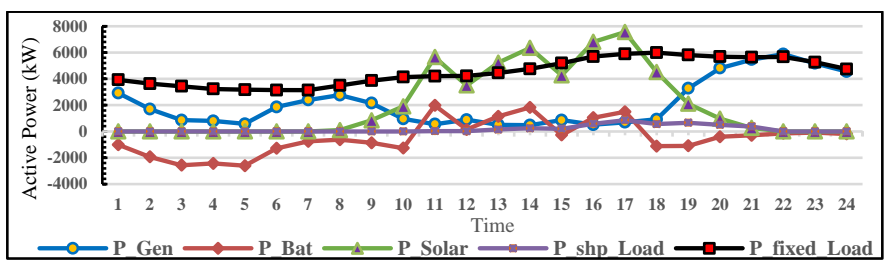

Fig. 13. Dispatch plan for the EPRI J1 test feeder generated by distributed consensus ADMM OPF algorithm.

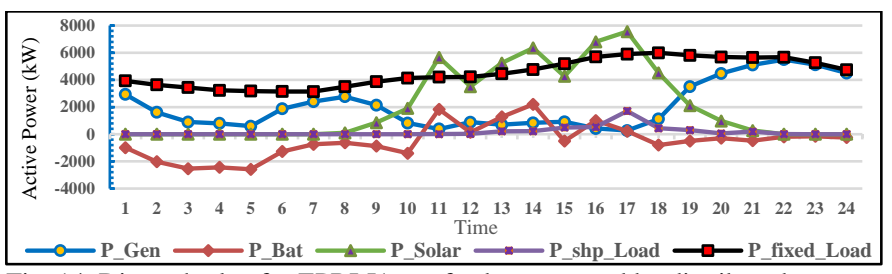

Fig. 14. Dispatch plan for EPRI J1 test feeder generated by distributed

consensus ADMM OPF algorithm implemented on the blockchain network.

We can observe from the dispatch plan that the battery energy storage responds to the peak demand and charges from the excess solar power. The charging schedule for the electric vehicles in the network coincides with solar power generation.

\section{Comparative Analysis}

TABLE 1 and TABLE 2 presents the comparison of centralized and distributed consensus ADMM OPF dispatch plan for the ACEP PSI Lab model with the lab test results. The generation and consumption are for a period of 1 hour. 
TABLE 1: ELECTRIC ENERGY GENERATION/CONSUMPTION OVER 1 HOUR FOR ACEP PSI LAB

\begin{tabular}{|c|c|c|c|c|c|}
\hline OPF Type & $\begin{array}{c}\text { Diesel Gen } \\
\text { (kWh) }\end{array}$ & $\begin{array}{c}\text { BESS Charge } \\
\text { (kWh) }\end{array}$ & $\begin{array}{c}\text { BESS Discharge } \\
\text { (kWh) }\end{array}$ & $\begin{array}{c}\text { Wind Gen. } \\
\text { (kWh) }\end{array}$ & $\begin{array}{c}\text { Fixed load } \\
\text { (kWh) }\end{array}$ \\
\hline Centralized & 101.356 & 5.517 & 14.482 & 46.183 & 156.5 \\
\hline Distributed Consensus ADMM & 104.435 & 5.517 & 11.399 & 46.183 & 156.5 \\
\hline $\begin{array}{c}\text { Distributed Consensus ADMM- } \\
\text { Blockchain Network }\end{array}$ & 104.435 & 5.517 & 11.399 & 46.183 & 156.5 \\
\hline ACEP PSI Lab Test Results & 111.707 & 6.237 & 5.093 & 53.742 & 162.33 \\
\hline
\end{tabular}

TABLE 2: COMPARISON OF OPF ALGORITHM DISPATCH PLAN WITH ACEP PSI LAB TEST RESULTS

\begin{tabular}{|c|c|c|c|}
\hline $\begin{array}{c}\text { Gen. Type } \\
\text { (kWh) }\end{array}$ & $\begin{array}{c}\text { Centralized OPF } \\
\text { Results-LAB Test (\% } \\
\text { Difference) }\end{array}$ & $\begin{array}{c}\text { Distributed Consensus } \\
\text { ADMM OPF Results- } \\
\text { LAB Test (\% } \\
\text { Difference) }\end{array}$ & $\begin{array}{c}\text { Distributed Consensus } \\
\text { ADMM OPF over } \\
\text { Blockchain Network } \\
\text { Results-LAB Test } \\
\text { (\% Difference) }\end{array}$ \\
\hline Diesel Generator & $9.72 \%$ & $6.73 \%$ & $6.73 \%$ \\
\hline BESS Charging & $12.25 \%$ & $12.25 \%$ & $12.25 \%$ \\
\hline BESS Discharging & $95.93 \%$ & $76.47 \%$ & $76.47 \%$ \\
\hline Wind Generation & $15.13 \%$ & $15.13 \%$ & $15.13 \%$ \\
\hline Fixed Load & $3.65 \%$ & $3.65 \%$ & $3.65 \%$ \\
\hline
\end{tabular}

We observe in these tables that there is a vast difference in the energy discharge from battery energy storage between the OPF algorithms dispatch plan and lab test results. This difference is because for the first $25 \mathrm{~min}$, the lab test was performed without the SRC strategy, and the loads were fed by the diesel generator instead of battery energy storage. The table also shows the difference in the wind energy forecast and the actual generation.

TABLE 3 \&TABLE 4 presents the comparison of distributed consensus ADMM OPF with the centralized algorithm for the IEEE 123-bus and EPRI J1 feeder test system. The generation and consumption are for 24 hours, i.e., day ahead.

TABLE 3: ELECTRIC ENERGY GENERATION/CONSUMPTION OVER 24 HOURS FOR IEEE 123-BUS and EPRI J1 TEST FEEDER

\begin{tabular}{|c|c|c|c|c|}
\hline System & OPF Type & $\begin{array}{c}\text { Diesel Gen. } \\
\text { (kWh) }\end{array}$ & $\begin{array}{c}\text { BESS Charge } \\
\mathbf{( k W h )}\end{array}$ & $\begin{array}{c}\text { BESS Discharge } \\
\mathbf{( k W h )}\end{array}$ \\
\hline \multirow{2}{*}{$\begin{array}{c}\text { IEEE 123-Bus } \\
\text { Test Feeder }\end{array}$} & Centralized & 19713.12 & 4725.729 & 8375.74 \\
\cline { 2 - 5 } & Distributed Consensus ADMM & 20000.25 & 4705.957 & 6983.39 \\
\cline { 2 - 5 } & $\begin{array}{c}\text { Distributed Consensus ADMM- } \\
\text { Blockchain Network }\end{array}$ & 20836.04 & 5528.73 & 7070.74 \\
\hline \multirow{2}{*}{$\begin{array}{c}\text { EPRI J1 Test } \\
\text { Feeder }\end{array}$} & Cistributed Consensus ADMM & 51600.26 & 18368.45 & 26842.4 \\
\cline { 2 - 5 } & $\begin{array}{c}\text { Distributed Consensus ADMM- } \\
\text { Blockchain Network }\end{array}$ & 50598.64 & 15899.41 & 27840.17 \\
\hline
\end{tabular}

TABLE 4: COMPARISON OF DISTRIBUTED AND CENTRALIZED OPF ALGORITHM FOR IEEE 123-BUS and EPRI J1 TEST FEEDER

\begin{tabular}{|c|c|c|c|c|}
\hline \multirow{2}{*}{ System } & $\begin{array}{c}\text { Gen. Type } \\
\text { (kWh) }\end{array}$ & $\begin{array}{c}\text { Centralized OPF- } \\
\text { Consensus ADMM } \\
\text { OPF } \\
\text { (\% Difference) }\end{array}$ & $\begin{array}{c}\text { Centralized OPF- } \\
\text { Consensus ADMM } \\
\text { OPF on Blockchain } \\
\text { Network } \\
\text { (\% Difference) }\end{array}$ & $\begin{array}{c}\text { Consensus ADMM - } \\
\text { Consensus ADMM } \\
\text { OPF on Blockchain } \\
\text { Network } \\
\text { (\% Difference) }\end{array}$ \\
\hline \multirow{2}{*}{$\begin{array}{c}\text { IEEE 123-Bus } \\
\text { Test Feeder }\end{array}$} & Diesel Generator & $1.45 \%$ & $5.54 \%$ & $4.093 \%$ \\
\cline { 2 - 5 } & BESS Charging & $0.42 \%$ & $15.66 \%$ & $16.08 \%$ \\
\hline \multirow{2}{*}{$\begin{array}{c}\text { EPRI J1 Test } \\
\text { Feeder }\end{array}$} & DESS Discharging & $18.13 \%$ & $16.89 \%$ & $1.24 \%$ \\
\cline { 2 - 5 } & Diesel Generator & $6.14 \%$ & $4.18 \%$ & $1.96 \%$ \\
\cline { 2 - 5 } & BESS Charging & $34.8 \%$ & $20.66 \%$ & $14.4 \%$ \\
\hline \multirow{2}{*}{ BESharging } & $3.02 \%$ & $3.65 \%$ & $6.35 \%$ \\
\hline
\end{tabular}

We can observe from TABLE 3 and TABLE 4 that the percentage difference in the energy generation by the diesel generator for all the OPF algorithms is negligible. Hence, the performance quality of the proposed distributed consensus ADMM with and w/o blockchain network is comparable to the standard centralized OPF algorithm.

\section{Computation Details}

For the ACEP PSI lab, IEEE 123-bus, and EPRI J1 test feeder, the centralized OPF problem is simulated in a Pythonbased, open-source optimization modeling language Pyomo, and the proposed distributed optimization algorithm is simulated using CVXPY. The simulations are run on Windows
10 PC with $3.40 \mathrm{GHz}$ Intel Core i7 processor and 16 GB RAM. The solver used and computation time is listed in TABLE 5

As seen from TABLE 5, the computation time to solve distributed consensus ADMM OPF problem is reduced compared to the centralized one for all three test system. Hence the algorithm is scalable and will be able to handle much larger system where centralized OPF will be computationally intensive.

For distributed consensus ADMM on a blockchain network, the time taken to validate the transactions is approximately $125 \mathrm{~s}$ for the ACEP PSI lab and 1500s for IEEE 123-bus and EPRI J1 test system. The validation time is significantly high because each node transacts with the network in a serial fashion one after the other. In practice on edge computing devices, these transactions would be performed in parallel; providing the speed up by the factor equivalent to the number of regions the power distribution network is partitioned and will be demonstrated in future work.

TABLE 5: OPF ALGORITHM COMPUTATION TIME AND SOLVER

\begin{tabular}{|c|c|c|c|c|c|}
\hline & & \multicolumn{4}{|c|}{ OPF Type } \\
\hline & & Centralized & $\begin{array}{l}\text { Distributed } \\
\text { (ADMM) }\end{array}$ & Dist & tributed (ADMM w/ Blockchain) \\
\hline \multicolumn{2}{|c|}{ Solver } & Gurobi & OSQP [40] & & OSQP \\
\hline \multirow{3}{*}{$\begin{array}{c}\text { Computation } \\
\text { Time } \\
\text { (seconds) }\end{array}$} & ACEP PSI Lab & 0.01199 & 0.0035 & 0.0035 & \begin{tabular}{|c|}
125 (Time taken to validate \\
transactions on Blockchain network)
\end{tabular} \\
\hline & $\begin{array}{c}\text { IEEE 123-Bus } \\
\text { Test Feeder } \\
\end{array}$ & 0.1237 & 0.071 & 0.071 & $\begin{array}{c}1500 \text { (Time taken to validate } \\
\text { transactions on Blockchain network) }\end{array}$ \\
\hline & $\begin{array}{l}\text { EPRI J1 Test } \\
\text { Feeder } \\
\end{array}$ & 3.41 & 0.145 & 0.145 & $\begin{array}{c}1500 \text { (Time taken to validate } \\
\text { transactions on Blockchain network) }\end{array}$ \\
\hline
\end{tabular}

The algorithm is a part of an application which is being developed to integrate with the open-source ADMS platform GridAPPS-D [41]. The GridAPPS-D will act as a contracts account to deploy and manage the smart contract on Ethereum network and demonstration will be presented in future work.

\section{CONCLUSION}

A consensus ADMM distributed optimization algorithm was proposed for OPF by partitioning the distribution network into sub-networks/regions. The IEEE 123-bus and EPRI J1 test feeder is partitioned into seven regions, and the information is exchanged between those regions instead of all the nodes. This reduces the computation time as well as the communication overhead, and the algorithm converges quickly. By comparing the results with the centralized algorithm, it can be concluded that the consensus ADMM distributed OPF algorithm converged quickly with reliable results. The proposed algorithm, when implemented on the blockchain network, takes more time to validate the nodes, but it eliminates the need for the central aggregator/controller since the smart contract deployed on the blockchain network acts as the virtual aggregator. The virtual aggregator will provide redundancy in centralized communication infrastructure and will eliminate the need for a central aggregator in distributed communication infrastructure. The virtual aggregator also manages the synchronization between the sub-networks/regions.

\section{ACKNOWLEDGMENT}

The contributions to this research work were achieved through the Grid Modernization Laboratory Consortium 
(GMLC). The GMLC was established as part of the U.S. Department of Energy's Grid Modernization Initiative (GMI) to accelerate the modernization of the U.S. electricity infrastructure. The views expressed in the article do not necessarily represent the views of the U.S. Department of Energy or the United States Government.

The authors would like to thank Dr. Ron Melton, and Dr. Kevin Schneider at Pacific Northwest National Laboratory, ACEP, and the University of Alaska Fairbanks for providing the resources and the data needed for this research work.

\section{REFERENCES}

[1] [Online]. Available: https://www.abbconversations.com/2019/02/distributed-grid-management-101-ders-arereshaping-the-power-grid/

[2] [Online]. https://www.rff.org/publications/explainers/renewables-101integrating-renewables/

[3] O. Alsac, J. Bright, M. Prais, B. Stott, "Further Development in LPbased Optimal Power Flow," IEEE Transactions on Power Systems, vol. 5, no. 3, pp. 697-711, Aug. 1990. DOI: 10.1109/59.65896

[4] Q. Peng and S. H. Low, "Distributed Optimal Power Flow Algorithm for Radial Networks, I: Balanced Single-phase Case," IEEE Transactions on Smart Grid, vol. 9, no. 1, pp. 111-121, 2018.

[5] R. Jabr, "Radial Distribution Load Flow using Conic Programming," IEEE Transactions on Power Systems, vol. 21, no. 3, pp. 1458-1469, 2006. DOI: 10.1109/TPWRS.2006.879234

[6] M. Farivar and S. H. Low, "Branch Flow Model: Relaxations and Convexification - Part I," IEEE Transactions on Power Systems, vol. 28, no. 3, pp. 2554-2563, 2013. DOI: 10.1109/TPWRS.2013.2255317

[7] M. Farivar and S. H. Low, "Branch Flow Model: Relaxations and Convexification - Part II," IEEE Transactions on Power Systems, vol. 28, no. 3, pp. 2564-2572, 2013. DOI: 10.1109/TPWRS.2013.2255318

[8] S. H. Low, "Convex Relaxation of Optimal Power Flow, I: Formulations and Relaxations," IEEE Transactions on Control of Network Systems, vol. 1, no. 1, pp. 15-27, 2014. DOI: 10.1109/TCNS.2014.2309732

[9] S. H. Low, "Convex Relaxation of Optimal Power Flow, II: exactness," IEEE Transactions on Control of Network Systems, vol. 1, no. 2, pp. 177-189, 2014. DOI: 10.1109/TCNS.2014.2323634

[10] A. Gabash and P. Li, "Active-Reactive Optimal Power Flow in Distribution Networks with Embedded Gen and Battery Storage," IEEE Transactions of Power Systems, vol. 27, no. 4, pp. 2026-2035, 2012.

[11] M. Nick, R. Cherkaoui, and M. Paolone, "Optimal Allocation of Dispersed Energy Storage Systems in Active Distribution Networks for Energy Balance and Grid Support," IEEE Transactions on Power Systems, vol. 29, no. 5, pp. 2300-2310, 2014.

[12] S. K. Jadhav, "Optimal Power Flow in WInd Farm Microgrid using Dynamic Programming," in IEEE International Conference on Emerging Trends and Innovations in Engineering and Technological Research, 2018. DOI: 10.1109/ICETIETR.2018.8529128

[13] M. A. Abdulgalil, A. M. Amin, M. Khalid, and M. AlMuhaini, "Optimal Sizing, Allocation, Dispatch, and Power Flow of Energy Storage Integrated with Distributed Gen Units and a Wind Farm," in IEEE PES Asia-Pacific Power and Energy Engineering Conference, 2018.

[14] C. Wang, L. Fang, L. Pan, and Y. Wang, "Optimal Dispatch of an Island M.G. Including Multiple Energy Storage System," in IEEE Conference on Energy Internet and Energy System Integration, 2018.

[15] M. Sufyan, C. Tan, N. Rahim, S. R. S. Raihan, and M. Muhammad, "Dynamic Economic Dispatch of Isolated Microgrid with Energy Storage using MIQP," in IEEE International Conference on Intelligent and Advanced System, 2018. DOI: 10.1109/ICIAS.2018.8540623

[16] A. Lam, B. Zhang, and D. N. Tse, "Distributed Algorithms for Optimal Power Flow," in IEEE Conference on Decision and Control, 2012

[17] N. Li, L. Chen and S. H. Low, "Demand Response in Radial Distribution Networks: Distributed Algorithm," in IEEE Conference on Signals, Systems, and Computers (ASILOMAR), 2012.
[18] E. Devane and I. Lestas, "Stability and Convergence of Distributed Algorithms for the OPF Problem," in IEEE Conference on Decision and Control, 2013. DOI: 10.1109/CDC.2013.6760329

[19] S. Boyd, N. Parikh, E. Chu, B. Peleato and J. Eckstein, "Distributed Optimization and Statistical Learning via the Alternating Direction Method of Multipliers," NOW publishers - Foundations and Trends in Machine Learning, vol. 3, no. 1, pp. 1-122, 2010. DOI: $10.1561 / 2200000016$

[20] E. Dall'Anese, H. Zhu, and G. B. Giannakis, "Distributed Optimal Power Flow for Smart Microgrids," IEEE Transactions on Smart Grid, vol. 4, no. 3, pp. 1464-1475, 2013. DOI: 10.1109/TSG.2013.2248175

[21] T. Erseghe, "Distributed Optimal Power Flow Using ADMM," IEEE Transactions on Power Systems, vol. 29, no. 5, pp. 2370-2380, September 2014. DOI: 10.1109/TPWRS.2014.2306495

[22] S. Magnusson, P. C. Weeraddana, and C. Fischione, "A Distributed Approach for the Optimal Power-Flow Problem Based on ADMM and Sequential Convex Approximations," IEEE Transactions on Control of Network Systems, vol. 2, no. 3, pp. 238-253, September 2015. DOI: 10.1109/TCNS.2015.2399192

[23] A. X. Sun, D. T. Pham and S. Ghosh, "Fully Decentralized A.C. Optimal Power Flow Algorithms," in IEEE PES General Meeting, Vancouver, BC, Canada, 2013. DOI: 10.1109/PESMG.2013.6672864

[24] E. Munsing, J. Mather, and S. Moura, "Blockchains for Decentralized Optimizations of Energy Resources in Microgrid Networks," in IEEE Conference on Control Technology and Applications, Hawaii, USA, August 2017. DOI: 10.1109/CCTA.2017.8062773

[25] J. Guo, G. Hug, and O.K. Tonguz, "On the Role of Communications Plane in Distributed Optimization of Power Systems," IEEE Transactions on Industrial Informatics, vol. 14, no. 7, pp. 2903-2913, July 2018. DOI: 10.1109/TII.2017.2774243

[26] [Online]. Available: https://iiot-world.com/industrial-iot/connectedindustry/internet-of-things-and-scada-is-one-going-to-replace-theother/.

[27] J. Mattila, "Industrial Blockchain Platforms: An Exercise in Use Case Development in the Energy Industry," ETLA Working Papers, p. 27, October 112016.

[28] [Online]. Available: https://www.ibm.com/blogs/internet-of-things/iotnew-blockchain-service/

[29] [Online]. Available: http://acep.uaf.edu/media/174758/ACEP-PSI2016-04-26-A-2-web.pdf.

[30] C. Shah and R. Wies, "Algorithms for Optimal Power Flow in Isolated Distribution Networks Using Different Battery Energy Storage Models," in IEEE Innovative Smart Grid Technologies, Washington D.C., 2020. DOI: 10.1109/ISGT45199.2020.9087717

[31] C. Eyisi, A. S. Al-Sumaiti, K. Turitsyn, and Q. Li, "Mathematical Models for Optimization of Grid-Integrated Energy Storage Systems: A Review," in IEEE 2019 North American Power Symposium (NAPS), Wichita, KS, USA, October 2019.

[32] M. Andoni et al., "Blockchain Technology in the Energy Sector: A Systemetic Review of Challenges and Opportunities," Elsevier Renewable and Sustainable Energy and Reviews, vol. 100, pp. 143-174, February 2019. DOI: 10.1016/j.rser.2018.10.014

[33] J. Mattila, "The Blockchain Phenomenon: The Disruptive Potential of Distributed Consensus Architectures," ETLA Working Paper, May 2016.

[34] "Ethereum," May 7 2020. [Online]. Available: https://ethereum.org/.

[35] $2016 . \quad$ [Online]. Available: https://solidity.readthedocs.io/en/v0.6.8/index.html.

[36] P. Merriam and J. Carver, "Readthedocs.io," 2018. [Online]. Available: https://web3py.readthedocs.io/en/stable/overview.html.

[37] [Online]. Available: https://dataport.pecanstreet.org/idq.

[38] J. Wang, et al. "Load Aggregation Methods for Quasi-Static Power Flow Analysis on High PV Penetration Feeder," in IEEE/PES Transmission and Distribution Conference and Exposition (T\&D), April 2018.

[39] EPRI Feeder J1, Distributed PV (DPV) Monitoring and Feeder Analysis. Available: dpv.epri.com

[40] B. Stellato et al., "OSQP: an operator splitting solver for quadratic programs," Mathematical Programming Computation,

[41] 2020. [Online]. Available: https://gridapps-d.org/. 